The University of San Francisco

USF Scholarship: a digital repository@ Gleeson Library |

Geschke Center

2005

\title{
Gallium Oxide and Dioxide: Investigation of the Ground and Low-Lying Electronic States via Anion Photoelectron Spectroscopy
}

Giovanni Meloni

University of San Francisco, gmeloni@usfca.edu

S M. Sheehan

D M. Neumark

Follow this and additional works at: http://repository.usfca.edu/chem_fac

Part of the Chemistry Commons

\section{Recommended Citation}

Meloni G, Sheehan SM, Neumark DM. Gallium oxide and dioxide: investigation of the ground and low-lying electronic states via anion photoelectron spectroscopy. J Chem Phys. 2005 Feb 15;122(7):074317.

This Article is brought to you for free and open access by the Chemistry at USF Scholarship: a digital repository @ Gleeson Library| Geschke Center. It has been accepted for inclusion in Chemistry Faculty Publications by an authorized administrator of USF Scholarship: a digital repository @ Gleeson Library $\mid$ Geschke Center. For more information, please contact repository@usfca.edu. 


\title{
Gallium oxide and dioxide: Investigation of the ground and low-lying electronic states via anion photoelectron spectroscopy
}

\author{
Giovanni Meloni, Sean M. Sheehan, and Daniel M. Neumark ${ }^{\mathrm{a})}$ \\ Department of Chemistry, University of California,, Berkeley, California 94720 andChemical Sciences \\ Division, Lawrence Berkeley National Laboratory, Berkeley, California 94720
}

(Received 4 November 2004; accepted 30 November 2004; published online 9 February 2005)

\begin{abstract}
The $\mathrm{GaO}$ and $\mathrm{GaO}_{2}$ molecules were investigated using negative ion photoelectron spectroscopy. All the photoelectron spectra showed vibrationally resolved progressions. With the aid of electronic structure calculations and Franck-Condon spectral simulations, different molecular parameters and energetics of $\mathrm{GaO}^{-} / \mathrm{GaO}$ and $\mathrm{GaO}_{2}^{-} / \mathrm{GaO}_{2}$ were determined, including the electron affinity of $\mathrm{GaO}$, the vibrational frequency of $\mathrm{GaO}^{-}$, and the term energy, spin-orbit splitting, and vibrational frequency for the first excited $A^{2} \Pi_{\Omega}$ state of $\mathrm{GaO}$. The $\mathrm{GaO}_{2}^{-}$photoelectron spectra comprised three bands assigned as transitions from the linear $X^{1} \Sigma_{g}^{+}$ground state of $\mathrm{GaO}_{2}^{-}$to three linear neutral states: the $A^{2} \Pi_{g}, B{ }^{2} \Pi_{u}$, and $C^{2} \Sigma_{u}^{+}$states. The symmetric stretch frequencies of the anion and three neutral states as well as the spin-orbit splitting of the neutral ${ }^{2} \Pi$ states were determined. Electronic structure calculations found the neutral lowest energy linear structure to be only $63 \mathrm{meV}$ higher than the neutral bent geometry. () 2005 American Institute of Physics. [DOI: 10.1063/1.1850470]
\end{abstract}

\section{INTRODUCTION}

Increasing scientific and societal interest in nanotechnology has motivated investigations of new materials from which nanostructures can be built. Gallium oxide, $\mathrm{Ga}_{2} \mathrm{O}_{3}$, is a candidate material for nanostructures because of its attractive bulk properties such as luminescence ${ }^{1}$ and conduction. ${ }^{2}$ Gallium oxide nanowires, ${ }^{3}$ nanosheets, ${ }^{4}$ and nanoribbons ${ }^{5}$ have been successfully synthesized. While much effort has been devoted to the characterization of these materials in the nanosize and bulk regimes, a systematic study of the electronic and molecular properties of smaller subunits has not been undertaken. In this paper, we explore the ground and low-lying electronic states of the $\mathrm{GaO}$ and $\mathrm{GaO}_{2}$ molecules using anion photoelectron (PE) spectroscopy, and compare the results with those of the better known $\mathrm{AlO}$ and $\mathrm{AlO}_{2}$ molecules.

Despite the fact that several experimental studies have been performed on the simplest gallium oxide molecule, $\mathrm{GaO}$, relatively little is known about its electronic spectroscopy. The only bands analyzed so far lie in the $300-420 \mathrm{~nm}$ region and were identified as the $B^{2} \Sigma^{+}-X^{2} \Sigma^{+}$transition. ${ }^{6-8}$ Several vibrational analyses of these bands have been carried out, ${ }^{6-11}$ the most recent of which ${ }^{11}$ give $\omega_{e}\left(X^{2} \Sigma^{+}\right)$and $\omega_{e}\left(B^{2} \Sigma^{+}\right)$values of 766.8 and $761.5 \mathrm{~cm}^{-1}$, respectively. There have, however, been some difficulties in the rotational analysis due to overlapping bands and the presence of double heads attributed to the different isotopes. In particular, there are discrepancies regarding the $r_{e}\left(X^{2} \Sigma^{+}\right)$values which range from 1.725 (Refs. 12 and 13) to $2.08 \AA .{ }^{14}$ Electronic structure calculations by Kovba and Topol, ${ }^{15}$ Petsalakis et al., ${ }^{16}$ and Gowtham, Costales, and Pandey ${ }^{17}$ report bond lengths of $1.688,1.647$, and $1.72 \AA$, respectively, closer to the lower experimental value of $1.725 \AA$. Gowtham et al. found the

${ }^{a)}$ Electronic mail: dneumark@berkeley.edu bond length in $\mathrm{GaO}^{-}$to be nearly the same as in $\mathrm{GaO}$, and calculated the electron affinity of $\mathrm{GaO}$ to be $2.56 \mathrm{eV}$.

No information is available on the electronic spectrum of $\mathrm{GaO}_{2}$. The $\mathrm{GaO}_{2}$ molecule has been experimentally investigated only via IR spectroscopy of matrix isolated species. ${ }^{18-21}$ Zehe et al. ${ }^{18}$ analyzed the spectra obtained by the reaction of $\mathrm{Ga}$ atoms with $\mathrm{O}_{2}$ in cryogenic matrices of $\mathrm{N}_{2}$ and $\mathrm{Ar}$ and found that the simplest product is the bent superoxide $\mathrm{Ga}^{+} \mathrm{O}_{2}^{-}$. They assigned the vibrational frequency of $1089.5 \mathrm{~cm}^{-1}$ to the $\nu_{1}\left(a_{1}\right)$ mode corresponding to the $\mathrm{O}-\mathrm{O}$ stretch, and frequencies of 380 and $285.5 \mathrm{~cm}^{-1}$ to the $\nu_{2}\left(a_{1}\right)$ and $\nu_{3}\left(b_{2}\right)$ modes corresponding to the symmetric and antisymmetric $\mathrm{Ga}-\mathrm{O}$ stretch, respectively. Serebrennikov, Osin, and Maltsev ${ }^{19}$ reproduced the results of Zehe et al. ${ }^{18}$ in a similar study of $\mathrm{Ga}$ atoms with $\mathrm{O}_{2}$ in an Ar matrix. Andrews and co-workers ${ }^{20,21}$ recorded the IR spectra of several gallium oxide species. They observed a band at $568.7 \mathrm{~cm}^{-1}$ and assigned it to the symmetric stretch of the $C_{2 v} \mathrm{Ga}\left(\mathrm{O}_{2}\right)^{-}$anion based on $a b$ initio calculations and isotopic shifts. Moreover, they assigned a band at $912.7 \mathrm{~cm}^{-1}$ to the antisymmetric stretch mode of the linear centrosymmetric OGaO molecule. Andrews $e t a l{ }^{21}$ also performed $a b$ initio calculations on the $\mathrm{GaO}_{2}^{-}$anion and determined it to have a linear and symmetric ${ }^{1} \Sigma_{g}^{+}$ground state, whereas the anion ${ }^{1} A_{1}$ bent structure is $\approx 3.7 \mathrm{eV}$ higher in energy than the linear ground state at the second-order Møller-Plesset (MP2) level of theory.

Archibong and St-Amant ${ }^{22}$ and $\mathrm{Bu}$, Chan, and Song ${ }^{23}$ performed computations up to the coupled cluster singles and doubles including a perturbational estimate of triples $[\operatorname{CCSD}(\mathrm{T})]$ level of theory and concluded that the neutral $\mathrm{GaO}_{2}$ lowest energy structure is bent with $\mathrm{a}^{2} A_{2}$ ground state. Very recently, Gowtham, Costales, and Pandey ${ }^{17}$ investigated small gallium oxide clusters, $\mathrm{Ga}_{m} \mathrm{O}_{n}(m, n=1,2)$ at the Becke three-parameter exchange functional with the Lee, Yang, and Parr correlation functional (B3LYP) level of 
theory. They found the lowest energy isomer of the neutral $\mathrm{GaO}_{2}$ to have a $C_{2 v}$ symmetry, the neutral linear isomer to lie $0.12 \mathrm{eV}$ above the bent structure, and the lowest energy isomer of the anion to be linear and centrosymmetric.

In this investigation, we report the first photodetachment $\mathrm{PE}$ experiments on $\mathrm{GaO}^{-}$and $\mathrm{GaO}_{2}^{-}$using photon energies of $5.821(213 \mathrm{~nm}), 4.661(266 \mathrm{~nm})$, and $3.493 \mathrm{eV}(355 \mathrm{~nm})$. We observed the lowest excited electronic state of $\mathrm{GaO}$ and transitions to three linear electronic states from linear $\mathrm{GaO}_{2}^{-}$. The PE spectra of $\mathrm{GaO}$ and $\mathrm{GaO}_{2}$ show vibrationally resolved features. Electronic structure calculations and Franck-Condon spectral simulations were performed yielding neutral and anion vibrational frequencies as well as geometric changes that occur upon photodetachment. We also obtained the electron affinity (EA) of $\mathrm{GaO}$ and adiabatic detachment energies (ADE) for three linear electronic states of $\mathrm{GaO}_{2}$.

\section{EXPERIMENT}

The experiments were carried out on a tandem time-offlight (TOF) negative ion PE spectrometer, incorporating a linear reflectron TOF mass spectrometer and a field-free TOF photoelectron analyzer. Details of this apparatus have been previously reported. ${ }^{24,25}$

$\mathrm{GaO}^{-}$and $\mathrm{GaO}_{2}^{-}$were generated by focusing the second harmonic $(532 \mathrm{~nm}, 10-15 \mathrm{~mJ} /$ pulse, $20 \mathrm{~Hz})$ of a pulsed Nd:YAG laser (YAG-yttrium aluminum garnet) onto a rotating and translating GaN disk. The disk was produced by pressing a $99.9 \%$ pure $\mathrm{GaN}$ powder (Aldrich) in an isostatic press at $200 \mathrm{kpsi}$. Although some $\mathrm{Ga}_{x} \mathrm{~N}_{y}^{-}$anions were seen (for which PE spectra will be reported shortly), the mass spectrum was dominated by oxides, most likely from surface oxidation of the target. The plume created by laser ablation was crossed perpendicularly by a pulse of $\mathrm{Ar}$ gas from a piezoelectric valve and expanded through a $19 \mathrm{~mm}$ long clustering channel. It was then skimmed and entered the first differentially pumped region of the linear reflectron TOF, where the anions formed during the expansion were extracted at $90^{\circ}$ from the molecular beam by a pulsed electric field and accelerated to $2.5 \mathrm{keV}$ beam energy. The mass resolution $m / \Delta m$ is $\approx 2000$. Each molecule has an isotopic distribution due to the natural isotopic abundance of Ga. In each case, the most intense mass peak was photodetached.

Mass-selected anions were photodetached with the third $(355 \mathrm{~nm}, 3.493 \mathrm{eV})$, fourth $(266 \mathrm{~nm}, 4.661 \mathrm{eV})$, or fifth harmonic $(213 \mathrm{~nm}, 5.821 \mathrm{eV})$ of a pulsed fixed-frequency $\mathrm{Nd}$ :YAG laser. Typically, a pulse energy of $1-6 \mathrm{~mJ}$ was used. The electron kinetic energy (eKE) distribution was determined by TOF analysis in a $1 \mathrm{~m}$ field-free flight tube. The eKE scale was calibrated using the known $\mathrm{PE}$ spectra of $\mathrm{O}_{2}^{-}$ at $355 \mathrm{~nm}, \mathrm{Cl}^{-}, \mathrm{Br}^{-}$, and $\mathrm{I}^{-}$at $266 \mathrm{~nm}$, and $\mathrm{Cl}^{-}$and $\mathrm{CN}^{-}$at $213 \mathrm{~nm}$. The energy resolution is $8-10 \mathrm{meV}$ at $0.65 \mathrm{eV}$ eKE and degrades as $(\mathrm{eKE})^{3 / 2}$ at higher energy.

All the PE spectra presented in this study are plotted as a function of the electron binding energy (eBE), defined as

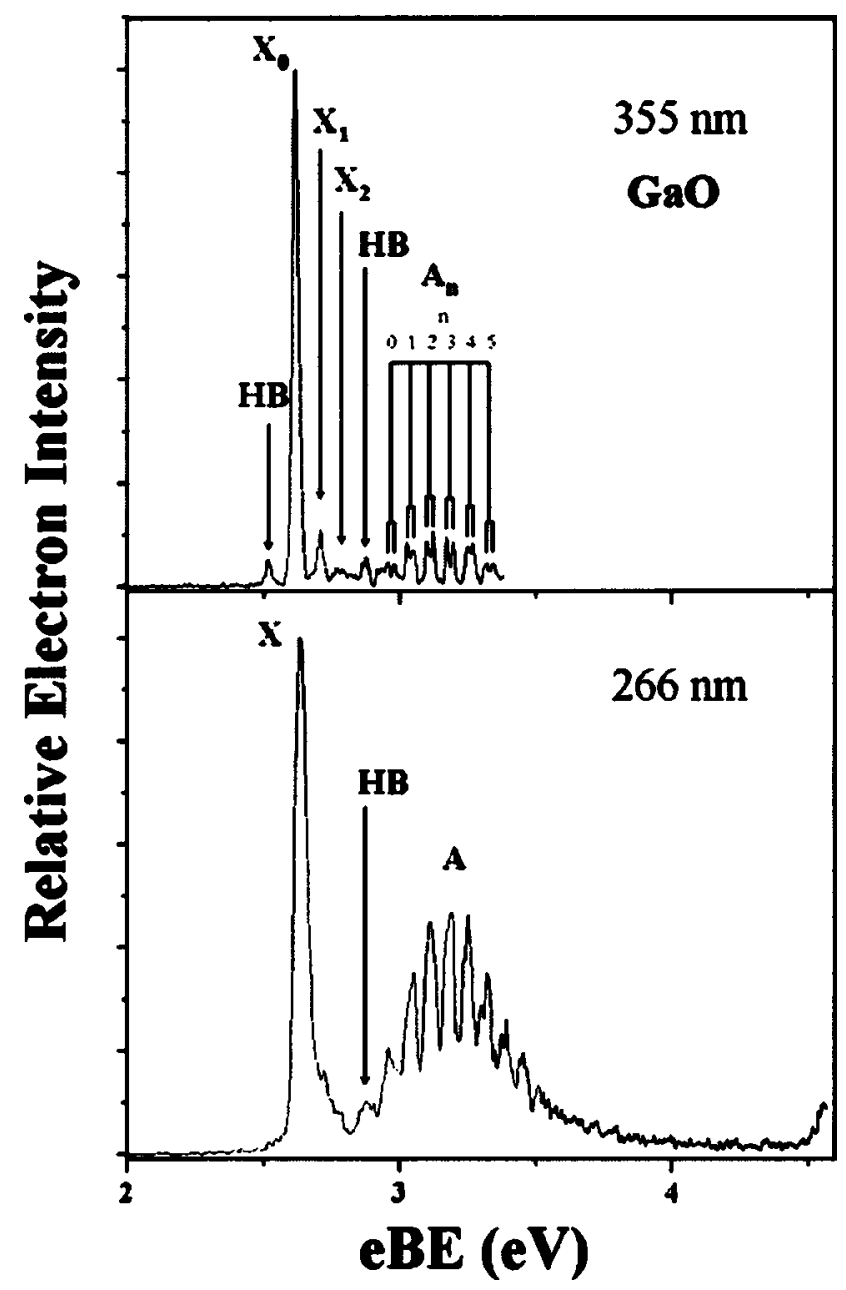

FIG. 1. Photoelectron spectra of $\mathrm{GaO}^{-}$at $355 \mathrm{~nm}$ (top) and $266 \mathrm{~nm}$ (bottom) photodetachment wavelengths taken with $\theta=90^{\circ}$.

$$
\mathrm{eBE}=h \nu-\mathrm{eKE}=\mathrm{EA}+E^{(0)}-E^{(-)},
$$

where $h \nu$ is the laser photodetachment energy $(3.493,4.661$, and $5.821 \mathrm{eV}), E^{(0)}$ is the internal energy of the neutral, and $E^{(-)}$the internal energy of the anion. The PE spectra were taken at two laser polarization angles, $0^{\circ}$ and $90^{\circ}$ with respect to the direction of electron detection. This angle was varied using a half-wave plate.

\section{RESULTS}

\section{A. Photoelectron spectra}

Figure 1 shows the $\mathrm{PE}$ spectra of $\mathrm{GaO}^{-}$taken at 355 and $266 \mathrm{~nm}$, the 266 and $213 \mathrm{~nm} \mathrm{PE}$ spectra of $\mathrm{GaO}_{2}^{-}$are displayed in Fig. 2. No variation of relative peak heights with laser polarization angle $\theta$ was observed; setting $\theta=90^{\circ}$ gave the highest electron signal. The 355 and $266 \mathrm{~nm}$ photoelectron spectra of $\mathrm{GaO}^{-}$comprise two distinct bands labeled as $X$ and $A$. The $266 \mathrm{~nm} \mathrm{GaO}^{-}$spectrum shows the same spectral features observed at $355 \mathrm{~nm}$ with a more extended progression of band $A$ and lower resolution because of the higher eKE. The $266 \mathrm{~nm} \mathrm{GaO}-\mathrm{PE}$ spectrum shows an intense band labeled $X$ and several smaller features, while the $213 \mathrm{~nm} \mathrm{GaO}-$ spectrum comprises three bands marked as $X$, $A$, and $B$. 


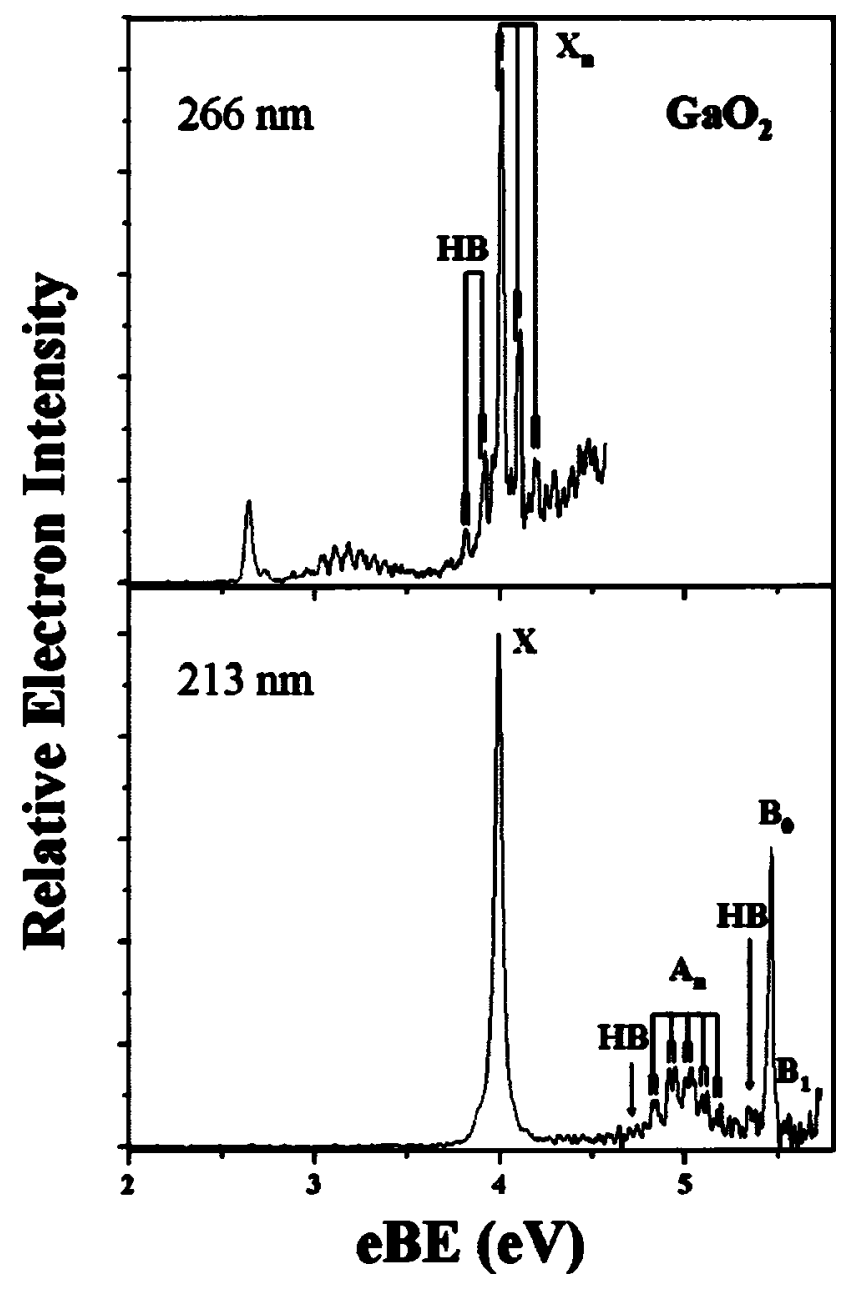

FIG. 2. Photoelectron spectra of $\mathrm{GaO}_{2}^{-}$at $266 \mathrm{~nm}$ (top) and $213 \mathrm{~nm}$ (bottom) photodetachment wavelengths taken with $\theta=90^{\circ}$.

In the $355 \mathrm{~nm} \mathrm{PE}$ spectrum of $\mathrm{GaO}^{-}$, the main band $X$, beginning at $2.61 \mathrm{eV}\left(X_{0}\right)$, shows a resolved vibrational progression with a peak spacing $\left(X_{0}-X_{1}\right)$ of $\approx 770 \mathrm{~cm}^{-1}$, very close to the literature value ${ }^{11}$ of $767 \mathrm{~cm}^{-1}$ for the fundamental vibrational frequency of the $X^{2} \Sigma^{+}$state. The short progression shows that the geometry change is small upon photodetachment. Band $A$ comprises a more extended vibrationally resolved progression starting at $2.97 \mathrm{eV}\left(A_{0}\right)$ with a peak spacing of $\approx 600 \mathrm{~cm}^{-1}$. Moreover, each peak of band $A$ seems to be a partially resolved doublet with a splitting of $160 \mathrm{~cm}^{-1}$. According to our density functional (DFT) calculations (see following section), the first excited electronic state of $\mathrm{GaO}$ is the $A^{2} \Pi$ state, while the $\mathrm{GaO}^{-}$anion is predicted to have a $X^{1} \Sigma^{+}$electronic ground state with a $a^{3} \Pi$ state lying at $\approx 2.9 \mathrm{eV}$ above the ground state. Consequently, we assign peak $X_{0}$ and $A_{0}$ to the vibrational origins of the $X^{2} \Sigma^{+} \leftarrow X^{1} \Sigma^{+}$and $A^{2} \Pi \leftarrow X^{1} \Sigma^{+}$electronic transitions. Since peak $X_{0}$ occurs at eBE $=2.95 \mathrm{eV}$, this is the approximate adiabatic EA of $\mathrm{GaO}$. Two smaller peaks are present in the $355 \mathrm{~nm}$ PE spectrum, labeled as $\mathrm{HB}$, at 2.51 and $2.87 \mathrm{eV}$ eBE, each lying $810 \mathrm{~cm}^{-1}$ from $X_{0}$ and $A_{0}$. They are assigned to anion vibrational hot bands, yielding an anion vibrational frequency of $810 \mathrm{~cm}^{-1}$.

In Fig. 2, the first two bands at lowest eBE in the $266 \mathrm{~nm}$ spectrum of $\mathrm{GaO}_{2}^{-}$appear at the same eBE as bands
$X$ and $A$ in the $\mathrm{GaO}^{-}$spectra and are not present in the $213 \mathrm{~nm} \mathrm{GaO}_{2}^{-} \mathrm{PE}$ spectrum. Indeed, when the $266 \mathrm{~nm} \mathrm{GaO}^{-}$ spectrum is superimposed to the $266 \mathrm{~nm} \mathrm{GaO}_{2}^{-}$spectrum, the bands perfectly match. The appearance of $\mathrm{GaO}^{-}$signal in the $\mathrm{GaO}_{2}^{-} \mathrm{PE}$ spectrum suggests the presence of a dissociative state of $\mathrm{GaO}_{2}^{-}$at $\approx 4.6 \mathrm{eV}$. Then, it is straightforward to assign the $\mathrm{GaO}^{-}$bands to a two photon process, according to which $\mathrm{GaO}^{-}$is produced by photodissociation of $\mathrm{GaO}_{2}^{-}$at $266 \mathrm{~nm}$ followed by photodetachment. No evidence for photodissociation of $\mathrm{GaO}_{2}^{-}$was seen at 213 or $355 \mathrm{~nm}$.

Band $X$ of the $266 \mathrm{~nm}$ spectrum, the first $\mathrm{GaO}_{2}$ feature, starts at $\approx 4 \mathrm{eV}$ and comprises a resolved vibrational progression with a peak spacing of $760 \mathrm{~cm}^{-1}$. Moreover, the peaks reveal partially resolved doublets with a splitting of $\approx 160 \mathrm{~cm}^{-1}$. The two small spectral features labeled as HB at $\mathrm{eBE}=3.82$ and $3.91 \mathrm{eV}$ are assigned as hot band transitions, yielding an approximate anion vibrational frequency of $770 \mathrm{~cm}^{-1}$. The $213 \mathrm{~nm}$ PE spectrum shows two additional bands, $A$ and $B$, beginning at 4.84 and $5.46 \mathrm{eV}$ eBE, respectively. Band $A$ has a resolved progression with a vibrational frequency of $700 \mathrm{~cm}^{-1}$, whereas band $B$ consists only of two peaks, $B_{0}$ and $B_{1}$, with a separation of $720 \mathrm{~cm}^{-1}$. Band $A$ also shows peaks with better-resolved doublets than band $X$, with a splitting of $220 \mathrm{~cm}^{-1}$. The full assignment of these bands is discussed in Sec. IV B.

\section{B. Electronic structure calculations}

In order to interpret the measured photoelectron spectra, we performed DFT calculations with the B3LYP method. The correlation consistent polarized valence triple- $\zeta$ (ccpVTZ) basis set was used for both Ga (Ref. 26) and O. ${ }^{27}$ The computations were carried out with the GAUSSIAN98 program suite. ${ }^{28}$ We calculated the energetics (including zero-point vibrational energies, term energies, and adiabatic detachment energies), optimized geometries, and harmonic vibrational frequencies for the ground and various excited electronic states of $\mathrm{GaO} / \mathrm{GaO}^{-}$and $\mathrm{GaO}_{2} / \mathrm{GaO}_{2}^{-}$.

Results are summarized in Table I. The electronic term energy $T_{e}$ is reported for each anion and neutral state. For each neutral state the ADE is also given, defined as the energy difference between the $v=0$ levels of the anion ground state and neutral electronic state in question.

The outer molecular orbital (MO) configuration for the $\mathrm{GaO}^{-} X \Sigma^{+}$ground state is found to be $4 \pi^{4} 10 \sigma^{2}$ from our calculations. The highest occupied molecular orbital (HOMO) $10 \sigma^{2}$ is antibonding, with Ga $4 s, \mathrm{Ga} 4 p$, and $\mathrm{O} 2 p$ character. The equilibrium bond length and harmonic vibrational frequency are found to be $1.715 \AA$ and $821 \mathrm{~cm}^{-1}$. The $a^{3} \Pi$ excited state, with an outer MO configuration of $10 \sigma^{1} 5 \pi^{1}$, lies $22930 \mathrm{~cm}^{-1}$ or $2.84 \mathrm{eV}$ above the ground state. The neutral $\mathrm{GaO}$ ground state has been experimentally characterized but some uncertainties persist regarding its bond length. Our optimized computed value of $1.717 \AA$ supports the earlier experimental determination of $1.725 \AA$ (Refs. 12 and 13) but not the most recent result of $2.08 \AA{ }^{14}$ The outer MO configurations of the $\mathrm{GaO} X^{2} \Sigma^{+}$and $A^{2} \Pi$ states are $4 \pi^{4} 10 \sigma^{1}$ and $4 \pi^{3} 10 \sigma^{2}$, respectively. The computed bond distance of $1.888 \AA$ for the $A^{2} \Pi$ state is slightly 
TABLE I. B3LYP/cc-pVTZ computed optimized molecular parameters $\left(r_{\mathrm{Ga}-\mathrm{O}}\right.$ and $r_{\mathrm{O}-\mathrm{O}}$ in angstrom, $\alpha$ in degrees, and $\nu$ in $\left.\mathrm{cm}^{-1}\right)$, zero-energy vibrational energies ( $\mathrm{ZPE}$ in $\mathrm{eV})$, term energies $\left(T_{e}\right.$ in $\left.\mathrm{cm}^{-1}\right)$, and adiabatic detachment energies (ADE in $\mathrm{eV}$ ).

\begin{tabular}{|c|c|c|c|c|c|c|c|c|c|c|}
\hline Molecule & State & $r_{\mathrm{Ga}-\mathrm{O}}$ & $r_{\mathrm{O}-\mathrm{O}}$ & $\alpha$ & $\nu_{1}^{\mathrm{a}}$ & $\nu_{2}$ & $\nu_{3}$ & $\mathrm{ZPE}$ & $T_{e}$ & $\mathrm{ADE}$ \\
\hline \multirow[t]{2}{*}{$\mathrm{GaO}^{-}$} & ${ }^{1} \Sigma^{+}$ & 1.715 & & & 827 & & & 0.051 & $\ldots$ & \\
\hline & ${ }^{3} \Pi$ & 1.786 & & & 591 & & & 0.037 & 22930 & \\
\hline \multirow[t]{2}{*}{$\mathrm{GaO}$} & ${ }^{2} \Sigma^{+}$ & 1.717 & & & 734 & & & 0.045 & $\ldots$ & 2.38 \\
\hline & ${ }^{2} \Pi$ & 1.888 & & & 562 & & & 0.035 & 1860 & 2.61 \\
\hline \multirow[t]{2}{*}{$\mathrm{GaO}_{2}^{-}$} & ${ }^{1} \Sigma_{g}^{+}$ & 1.691 & & 180 & 206 & 775 & 930 & 0.132 & $\ldots$ & \\
\hline & ${ }^{1} A_{1}^{8}$ & 1.901 & 1.550 & 48 & 435 & 506 & 824 & 0.110 & 15880 & \\
\hline \multirow[t]{5}{*}{$\mathrm{GaO}_{2}$} & ${ }^{2} \mathrm{~A}_{2}$ & 2.080 & 1.340 & 38 & 286 & 389 & 1173 & 0.115 & $\ldots$ & \\
\hline & ${ }^{2} \Pi_{g}$ & 1.695 & & 180 & 185 & 757 & 661 & 0.111 & 510 & 3.56 \\
\hline & ${ }^{2} \Pi_{u}^{g}$ & 1.725 & & 180 & 158 & 657 & 1133 & 0.130 & 8920 & 4.61 \\
\hline & ${ }^{2} \Sigma_{u}^{+}$ & 1.701 & & 180 & 167 & 724 & 819 & 0.116 & 11760 & 4.99 \\
\hline & ${ }^{2} A_{1}^{u}$ & 1.814 & 1.624 & 53 & 576 & 555 & 792 & 0.119 & 13960 & \\
\hline
\end{tabular}

${ }^{\mathrm{a}}$ For linear molecules $\nu_{1}$ is the bending $\pi, \nu_{2}$ the symmetric stretching $\sigma_{g}$, and $\nu_{3}$ the antisymmetric stretching $\sigma_{u}$ mode; for nonlinear molecules $\nu_{1}$ is the antisymmetric stretching $b_{2}, \nu_{2}$ the symmetric stretching $a_{1}$, and $\nu_{3} a_{1}$ the bending mode.

longer than the previously calculated values of $1.804 \AA$ (Ref. 16) and $1.836 \AA .{ }^{15}$ The calculated term value $T_{e}\left(A^{2} \Pi\right)$ is $1860 \mathrm{~cm}^{-1}$ or $0.23 \mathrm{eV}$, close to the splitting between the apparent origins of bands $X$ and $A$ in the PE spectrum of $\mathrm{GaO}^{-}$. The harmonic vibrational frequencies of 827,734 , and $562 \mathrm{~cm}^{-1}$ for the anion ground state, the neutral ground state, and first excited electronic state, respectively, are in very good agreement with the values obtained from the PE spectra.

Our B3LYP/cc-pVTZ calculations of $\mathrm{GaO}_{2}^{-}$yield a similar ordering of states as the work of Andrews et al., ${ }^{21}$ with the ${ }^{1} \Sigma_{g}^{+}$centrosymmetric geometry as the ground state. The $\mathrm{GaO}_{2}^{-} \sum_{g}^{+} 1$ outer MO configuration is $12 \sigma_{u}^{2} 4 \pi_{u}^{4} 5 \pi_{g}^{4}$. The $5 \pi_{g}^{4}$ HOMO has antibonding character, and it is composed primarily of linear combinations of $\mathrm{Ga} 3 d$ and $\mathrm{O} 2 p$ orbitals. The $\mathrm{GaO}_{2}^{-} V$-shape structure with an apex angle of $48^{\circ}$ lies $15880 \mathrm{~cm}^{-1}$ or $\approx 2 \mathrm{eV}$ above the $X^{1} \Sigma_{g}^{+}$state. On the other hand, the situation is inverted for neutral $\mathrm{GaO}_{2}$ for which the ground state has a bent structure $\left({ }^{2} A_{2}\right)$ and the first excited state $\left({ }^{2} \Pi_{g}\right)$ is linear. Our findings for the bent ${ }^{2} A_{2}$ state of $\mathrm{GaO}_{2}$ are similar to previous theoretical calculations. ${ }^{17,22,23}$ According to our calculations the $A^{2} \Pi_{g}$ state lies only $510 \mathrm{~cm}^{-1}$ or $63 \mathrm{meV}$ above the ground state, and has an outer MO configuration of $12 \sigma_{u}^{2} 4 \pi_{u}^{4} 5 \pi_{g}^{3}$. Based only on these DFT energetics, we cannot rule out the possibility that the global minimum of $\mathrm{GaO}_{2}$ is indeed linear. We find $r_{\mathrm{Ga}-\mathrm{O}}$ for the $A^{2} \Pi_{g}$ state to be $1.695 \AA$, and the symmetric stretch of $757 \mathrm{~cm}^{-1}$ agrees very well with the experimental peak spacing of $760 \mathrm{~cm}^{-1}$ for band $X$. The $B^{2} \Pi_{u}$ and $C^{2} \Sigma_{u}^{+}$electronic states lie $8410 \mathrm{~cm}^{-1}(1.043 \mathrm{eV})$ and $11250 \mathrm{~cm}^{-1}(1.395 \mathrm{eV})$ above the $A^{2} \Pi_{g}$ state, close to the spacings between the origins of bands $X, A$, and $C$.

\section{DISCUSSION}

Assignment of the electronic bands and vibrational progressions is facilitated by comparison with the molecular parameters and energetics provided by the electronic structure calculations described in the preceding section. In addition, the B3LYP/cc-pVTZ calculations supplied the force con- stants necessary for simulating the PE spectra within the Franck-Condon (FC) approximation. For $\mathrm{GaO}^{-}$, the electronic structure calculations indicate that bands $X$ and $A$ in the PE spectra should be assigned as transitions to the neutral $X^{2} \Sigma^{+}$and $A^{2} \Pi$ states, respectively. The calculations suggest that in the $\mathrm{GaO}_{2}^{-} \mathrm{PE}$ spectra, bands $X, A$, and $B$ are from transitions from the linear anion to the three low-lying $A^{2} \Pi_{g}$, $B^{2} \Pi_{u}$ and $C^{2} \Sigma_{u}^{+}$linear neutral states. FC simulations of the $\mathrm{GaO}_{2}^{-}$spectra were carried out within the parallel-mode approximation assuming harmonic oscillator potentials. Starting from the force constants and computed parameters obtained from the calculations for the initial state of the anion and the final state of the neutral, the normal coordinate changes, term energies, and vibrational frequencies were used as input for the simulations and successively optimized to reproduce the experimental PE spectra. Transition origins were taken from experiment, as well as the experimentally known parameters. The anion vibrational temperature was assumed to be $1000 \mathrm{~K}$ for both $\mathrm{GaO}^{-}$and $\mathrm{GaO}_{2}^{-}$. The parameters used in the PE spectra simulations are listed in Table II, and the simulated spectra for $\mathrm{GaO}$ and $\mathrm{GaO}_{2}$ are shown in Fig. 3. Rotational broadening of the peaks is negligible because of the small rotational constants for $\mathrm{GaO} / \mathrm{GaO}^{-}$and $\mathrm{GaO}_{2} / \mathrm{GaO}_{2}^{-}$.

\section{A. $\mathrm{GaO}$}

One-electron photodetachment from the two highest lying molecular orbitals of $\mathrm{GaO}^{-}$results in the neutral $X^{2} \Sigma^{+}$ $\left(4 \pi^{4} 10 \sigma^{1}\right)$ ground and $A^{2} \Pi\left(4 \pi^{3} 10 \sigma^{2}\right)$ excited electronic states. In simulations of the PE spectra, the known neutral ground state bond distance ${ }^{12,13}$ and vibrational frequency ${ }^{11}$ were fixed at $1.725 \AA$ and $767 \mathrm{~cm}^{-1}$, whereas the molecular parameters of the anion ground state, the neutral first excited state, and $\mathrm{EA}(\mathrm{GaO})$ were varied to achieve best fit.

The FC simulation of band $X$ for the $355 \mathrm{~nm}$ PE spectrum yields the adiabatic $\mathrm{EA}(\mathrm{GaO})$ as $2.612 \pm 0.008 \mathrm{eV}$, $0.23 \mathrm{eV}$ higher than our computed value of $2.38 \mathrm{eV}$, but very close to the value of $2.56 \mathrm{eV}$ calculated by Gowtham, Costales, and Pandey. ${ }^{17}$ Band $A$, assigned to the $A^{2} \Pi \leftarrow X^{1} \Sigma^{+}$ 
TABLE II. Optimized parameters used in the FC simulation of the PE spectra. The vibrational modes $\nu$ and the spin-orbit constants $A_{e}$ are in $\mathrm{cm}^{-1}$, the equilibrium bond distance change $\Delta r_{e}$ in angstrom, the $\mathrm{ADE}$ in $\mathrm{eV}$, the term energies $T_{e}$ in $\mathrm{cm}^{-1}$, and the anion vibrational temperature $T$ in $\mathrm{K}$.

\begin{tabular}{cccccccccc}
\hline \hline Molecule & Band & State & $\nu^{(-)}$ & $\nu^{(0)}$ & $\left|A_{e}\right|$ & $\Delta r_{e}$ & ADE & $T_{e}$ \\
\hline \multirow{2}{*}{$\mathrm{GaO}$} & $X$ & ${ }^{2} \Sigma^{+}$ & 805 & 767 & & 0.025 & $2.612 \pm 0.008$ & $\ldots$ \\
& $A$ & ${ }^{2} \Pi$ & 805 & 580 & 170 & 0.174 & $2.965 \pm 0.008$ & 2850 & 1000 \\
$\mathrm{GaO}_{2}$ & $X$ & ${ }^{2} \Pi_{g}$ & 770 & 760 & 160 & 0.028 & $4.008 \pm 0.008$ & $\ldots$ & 1000 \\
& $A$ & ${ }^{2} \Pi_{u}$ & 770 & 710 & 210 & 0.069 & $4.839 \pm 0.010$ & 6700 & 1000 \\
& $B$ & ${ }^{2} \Sigma_{u}^{+}$ & 770 & 720 & & 0.018 & $5.464 \pm 0.008$ & 11740 \\
\hline \hline
\end{tabular}

electronic transition, shows a resolved vibrational progression yielding a frequency of $580 \pm 30 \mathrm{~cm}^{-1}$ for the $A^{2} \Pi$ state. Each vibrational peak comprising band $A$ presents a partially resolved doublet that provides the determination of the spinorbit splitting between the ${ }^{2} \Pi_{3 / 2}$ and ${ }^{2} \Pi_{1 / 2}$ states as $170 \pm 30 \mathrm{~cm}^{-1}$. In addition, from the band $A$ vibronic origin, we derived the $A^{2} \Pi$ term energy as $2850 \pm 90 \mathrm{~cm}^{-1}$ or $0.353 \pm 0.011 \mathrm{eV}$.

The two smaller spectral features labeled as HB in the $355 \mathrm{~nm}$ spectrum are transitions from vibrationally excited levels of $\mathrm{GaO}^{-}$. The spacing between the $\mathrm{HB}$ peaks and the vibronic origins of bands $X$ and $A$ provides the anion ground state frequency as $805 \pm 30 \mathrm{~cm}^{-1}$. From the spectral simulations, we also obtain the absolute values of the bond distance change for the $X^{2} \Sigma^{+} \leftarrow X^{1} \Sigma^{+}$and $A^{2} \Pi \leftarrow X^{1} \Sigma^{+}$electronic transitions, as 0.025 and $0.174 \AA$. If we assume the signs of these optimized values of $\Delta r_{\mathrm{Ga}-\mathrm{O}}$ to be the same as the signs for the calculated $\Delta r_{\mathrm{Ga}-\mathrm{O}}$ values from Table I, we find equilibrium bond lengths of $1.70 \pm 0.01$ and $1.87 \pm 0.01 \AA$ for the

\section{$\mathrm{GaO}$}
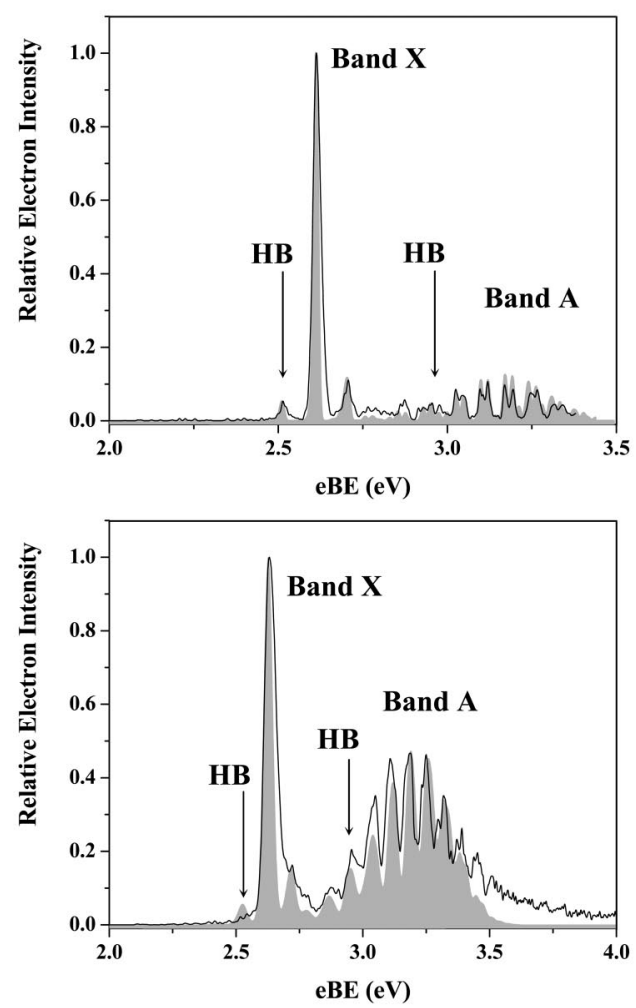

$X^{1} \Sigma^{+}$anion ground state and neutral $A^{2} \Pi$ state, respectively, in close agreement with the calculated bond lengths in Table I.

The PE spectrum of $\mathrm{GaO}^{-}$is remarkably similar to the $\mathrm{AlO}^{-} \mathrm{PE}$ spectrum measured by Desai, Wu, and Wang. ${ }^{29}$ The $\mathrm{AlO}^{-}$spectrum also comprises two bands with the same state assignments as in the $\mathrm{GaO}^{-}$spectrum: a very short progression, assigned to the $X^{2} \Sigma^{+} \leftarrow X^{1} \Sigma^{+}$transition, and a more extended band assigned to the $A^{2} \Pi \leftarrow X^{1} \Sigma^{+}$transition. Hence, the state orderings are the same, and changes in geometry upon photodetachment are similar. Remarkably, the electron affinities of $\mathrm{GaO}$ and $\mathrm{AlO}$ are almost identical: 2.612 and $2.60 \mathrm{eV}$, respectively. The vibrational frequencies for $\mathrm{AlO}^{-}$and the $\mathrm{AlO}$ ground state ${ }^{30}$ are higher by $95 \mathrm{~cm}^{-1}$ and $212 \mathrm{~cm}^{-1}$, respectively, than the $\mathrm{GaO}^{-}$and $\mathrm{GaO}$ frequencies. The term value for the $A^{2} \Pi$ state in $\mathrm{GaO}, 0.353 \mathrm{eV}$, is about half the term value for the analogous state in $\mathrm{AlO}$, $0.66 \mathrm{eV}$. The spin-orbit splitting of $128.7 \mathrm{~cm}^{-1}$ for the $\mathrm{AlO}$ $A^{2} \Pi$ state $^{30}$ is not resolved in the PE spectra of Desai, Wu,
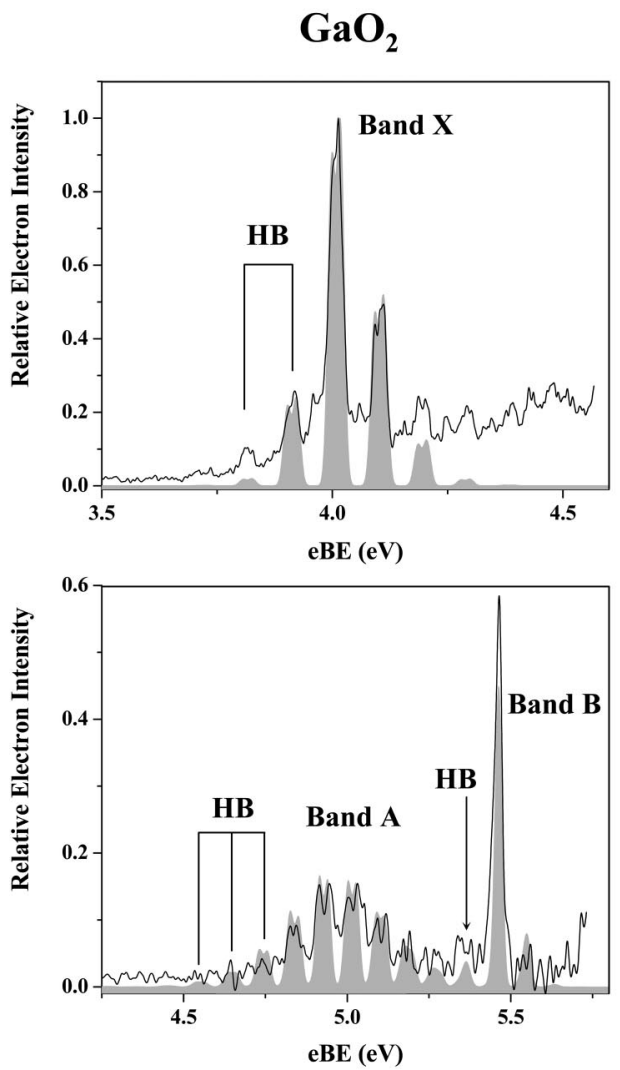

FIG. 3. Franck-Condon spectral simulations of $\mathrm{GaO}^{-}$and $\mathrm{GaO}_{2}^{-} \mathrm{PE}$ spectra (gray area) superimposed on the experimental spectra. 
and Wang ${ }^{29}$ and is comparable to the resolved splitting of $170 \mathrm{~cm}^{-1}$ for $\mathrm{GaO}$ determined in this investigation.

\section{B. $\mathrm{GaO}_{2}$}

The computational results described in Sec. III B, together with other theoretical studies ${ }^{21-23}$ on the $\mathrm{GaO}_{2}^{-} / \mathrm{GaO}_{2}$ molecules, indicate that the neutral and anion have different ground state geometries: the anion is linear and the neutral bent. At the B3LYP/cc-pVTZ level of theory, the outer MO configuration of the $\mathrm{GaO}_{2}^{-}$ground state is $12 \sigma_{u}^{2} 4 \pi_{u}^{4} 5 \pi_{g}^{4}$, and those of the neutral $A^{2} \Pi_{g}, B^{2} \Pi_{u}$, and $C^{2} \Sigma_{u}^{+}$states are $12 \sigma_{u}^{2} 4 \pi_{u}^{4} 5 \pi_{g}^{3}, 12 \sigma_{u}^{2} 4 \pi_{u}^{3} 5 \pi_{g}^{4}$, and $12 \sigma_{u}^{1} 4 \pi_{u}^{4} 5 \pi_{g}^{4}$, respectively. Since photodetachment is vertical, the transition between the linear anion and strongly bent (apex angle of $38^{\circ}$ ) ${ }^{2} A_{2}$ state would be very weak, so we only observe transitions to the three neutral linear states. We thus cannot tell from the PE spectra whether the $\mathrm{GaO}_{2}$ ground state is bent or linear; assuming our calculated energy ordering of the neutral states is correct, we can only obtain ADEs for the three linear states and not the electron affinity of $\mathrm{GaO}_{2}$.

The best-fit FC simulations of the $\mathrm{GaO}_{2}^{-} \mathrm{PE}$ spectra are shown in Fig. 3, and the optimized parameters used in these simulations are listed in Table II. All three bands show resolved vibrational structure. The FC analysis of band $X$ indicates that $\operatorname{ADE}\left(A^{2} \Pi_{g}\right)$ is $4.008 \pm 0.008 \mathrm{eV}$. This band comprises a progression in the totally symmetric mode $\sigma_{g}$ with a frequency of $760 \pm 30 \mathrm{~cm}^{-1}$, which is in perfect agreement with the computed B3LYP/cc-pVTZ value. The normal mode displacement of $0.160 \mathrm{amu}^{1 / 2} \AA$ for this mode needed to fit the spectrum corresponds to $\left|\Delta r_{\mathrm{Ga}-\mathrm{O}}\right|=0.03 \pm 0.01 \AA$ upon photodetachment, somewhat larger than the calculated change of $0.004 \AA$. In addition, each vibrational peak shows a partially resolved splitting that is assigned to the spin-orbit coupling of the $\Omega=1 / 2$ and $3 / 2$ components of the ${ }^{2} \Pi_{g}$ state, yielding a spin-orbit splitting of $160 \pm 30 \mathrm{~cm}^{-1}$.

The vibronic origin of band $A$ provides the $\operatorname{ADE}\left(B^{2} \Pi_{u}\right)$ of $4.839 \pm 0.010 \mathrm{eV}$ and yields an energy separation between the neutral ${ }^{2} \Pi_{g}$ and ${ }^{2} \Pi_{u}$ states of $6700 \pm 105 \mathrm{~cm}^{-1}$ or $0.831 \pm 0.013 \mathrm{eV}$. The computed ${ }^{2} \Pi_{g^{-}}{ }^{2} \Pi_{u}$ separation overestimates the experimental value by $\approx 25 \%$. Band $A$ also presents a vibrational resolved progression, more extended than band $X$, that is fit using the totally symmetric stretching mode $\sigma_{g}$ of $710 \pm 30 \mathrm{~cm}^{-1}$. The calculated frequency is $53 \mathrm{~cm}^{-1}$ lower than the best-fit value. The $\Delta Q$ of $0.390 \mathrm{amu}^{1 / 2} \AA$ for this vibrational mode results in a $\left|\Delta r_{\mathrm{Ga}-\mathrm{O}}\right|$ of $0.07 \pm 0.01 \AA$. The B3LYP/cc-pVTZ $\left|\Delta r_{\mathrm{Ga}-\mathrm{O}}\right|$ value for the $A^{2} \Pi_{g} \leftarrow X^{1} \Sigma_{g}^{+}$transition is $0.034 \AA$. As for band $X$, each vibrational peak of band $A$ is split by $210 \pm 30 \mathrm{~cm}^{-1}$, because of the spin-orbit coupling in the ${ }^{2} \Pi_{u}$ state.

From the FC simulation of band $B$, we derive the $\operatorname{ADE}\left(C^{2} \Sigma_{u}^{+}\right)$of $5.464 \pm 0.008 \mathrm{eV}$, yielding an energy separation between the neutral $A^{2} \Pi_{g}$ and $C^{2} \Sigma_{u}^{+}$states of $11740 \pm 90 \mathrm{~cm}^{-1}$ or $1.456 \pm 0.011 \mathrm{eV}$. The calculated ${ }^{2} \Pi_{g}$ $-{ }^{2} \Sigma_{u}^{+}$separation underestimates the experimental finding by only $4 \%$. The small vibrational excitation for band $B$ denotes a small geometry change upon removing the $12 \sigma_{u}$ electron. From the FC simulation, we derive the totally symmetric stretching mode of $720 \pm 30 \mathrm{~cm}^{-1}$ with an associated $\left|\Delta r_{\mathrm{Ga}-\mathrm{O}}\right|$ of $0.02 \pm 0.01 \AA$ for the ${ }^{2} \Sigma_{u}^{+}$state. The computed frequency is in excellent agreement with the best-fit result, whereas the calculated $\Delta r_{\mathrm{Ga}-\mathrm{O}}$ is $0.01 \AA$. No doublet splitting was observed for band $B$, consistent with our assignment of the ${ }^{2} \Sigma_{u}^{+}$ state.

All the three bands show smaller spectral features labeled as HB that are assigned to transitions from vibrationally excited levels of $\mathrm{GaO}_{2}^{-}$. The spacing between the $\mathrm{HB}$ peaks and the vibronic origins of bands $X, A$, and $B$ provides the first experimental determination of the anion symmetric stretching mode of $770 \pm 30 \mathrm{~cm}^{-1}$, in agreement with the calculated value of $775 \mathrm{~cm}^{-1}$.

Finally, we compare the findings for $\mathrm{GaO}_{2}^{-} / \mathrm{GaO}_{2}$ with those for the isoelectronic $\mathrm{AlO}_{2}^{-} / \mathrm{AlO}_{2}$ molecules. The $\mathrm{AlO}_{2}^{-}$ PE spectrum obtained by Desai et al. ${ }^{31}$ using $6.42 \mathrm{eV}$ photon energy looks very similar to the $5.821 \mathrm{eV} \mathrm{PE}$ spectrum of $\mathrm{GaO}_{2}^{-}$. In both cases, three bands are observed and assigned to linear $\leftarrow$ linear electronic transitions. From theoretical calculations ${ }^{32}$ it is found that the neutral $\mathrm{AlO}_{2}$ has two almost isoenergetic isomers with the linear geometry being the global minimum. On the other hand, from our computations and other calculations ${ }^{21-23}$ the lowest energy structure of the neutral $\mathrm{GaO}_{2}$ appears to be bent with an apex angle of $38^{\circ}$. The $\mathrm{AlO}_{2}^{-}$and $\mathrm{GaO}_{2}^{-} \mathrm{PE}$ spectra both show a high ADE for the first band, $4.23 \mathrm{eV}$ for $\mathrm{AlO}_{2}^{-}$and $4.008 \mathrm{eV}$ for $\mathrm{GaO}_{2}^{-}$. Also, the energy separation of the two excited electronic states relative to the first band is similar in the case of $\mathrm{AlO}_{2}$ and $\mathrm{GaO}_{2} ; \quad T_{e}\left(A^{2} \Pi_{u}\right)$ and $T_{e}\left(B^{2} \Sigma_{u}^{+}\right)$for $\mathrm{AlO}_{2}$ are $(0.65 \pm 0.03)$ and $(0.85 \pm 0.03) \mathrm{eV}$, respectively.

\section{CONCLUSIONS}

Vibrationally resolved anion photoelectron spectra of $\mathrm{GaO}^{-}$and $\mathrm{GaO}_{2}^{-}$are presented. These spectra show transitions to the ground and excited electronic states of the neutrals that were not previously observed. They are discussed in detail and analyzed with the aid of DFT calculations and FC simulations. In the $\mathrm{GaO}^{-} \mathrm{PE}$ spectra, bands $X$ and $A$ are assigned to transitions from the anion ground state to the neutral ground and first excited state, yielding the first experimental determination of $\mathrm{EA}(\mathrm{GaO})$ and $T_{e}\left(A^{2} \Pi\right)$. The vibrational frequencies for the anion ground state and the neutral first excited state were also obtained. Moreover, the resolved splitting of band $A$ allowed the determination of the spin-orbit coupling of the $A^{2} \Pi$ state. The $\mathrm{GaO}_{2}^{-} \mathrm{PE}$ spectrum shows three bands $X, A$, and $B$ that also present resolved vibrational structure. These bands are assigned to linear $\leftarrow$ linear electronic transitions. From the electronic structure calculations, we ascertained that the neutral $\mathrm{GaO}_{2}$ has two almost isoenergetic isomers, with the bent structure being the global minimum at only $63 \mathrm{meV}$ below the linear structure. From the FC analysis, we obtained the values of the symmetric stretching modes of the anion ground state, and three neutral linear excited electronic states, together with the bond distance changes for these transitions. Energy separations of the $B^{2} \Pi_{u}$ and $C^{2} \Sigma_{u}^{+}$relative to the first excited state $A^{2} \Pi_{g}$ were derived from the experimental ADE values. We did not observe the bent $\leftarrow$ linear electronic transition because of the very different geometries of the linear anion and 
bent neutral with and apex angle of $38^{\circ}$. Consequently, we were not able to resolve the issue of the neutral ground state geometry. Finally, we compared our experimental findings with those of the isoelectronic $\mathrm{AlO}^{-} / \mathrm{AlO}$ and $\mathrm{AlO}_{2}^{-} / \mathrm{AlO}_{2}$ molecules.

\section{ACKNOWLEDGMENT}

The authors gratefully acknowledge the financial support by the National Science Foundation under Grant No. DMR0139064.

${ }^{1}$ L. Binet and D. Gourier, J. Phys. Chem. Solids 59, 1241 (1998).

${ }^{2}$ Z. Hajnal, J. Miro, G. Kiss, F. Reti, P. Deak, R. C. Herndon, and J. M. Kuperberg, J. Appl. Phys. 86, 3792 (1999).

${ }^{3}$ S. Sharma and M. K. Sunkara, J. Am. Chem. Soc. 124, 12288 (2002).

${ }^{4}$ R. Z. Ma and Y. Bando, Chem. Phys. Lett. 367, 219 (2003).

${ }^{5}$ L. Fu, Z. M. Liu, Y. Q. Liu, B. X. Han, J. Q. Wang, P. G. Hu, L. C. Cao, and D. B. Zhu, J. Phys. Chem. B 108, 13074 (2004).

${ }^{6}$ M. L. Guernsey, Phys. Rev. 46, 114 (1934).

${ }^{7}$ V. Raziunas, S. Katz, and G. J. Macur, J. Chem. Phys. 39, 1161 (1963).

${ }^{8}$ V. Raziunas, G. J. Macur, and S. Katz, J. Chem. Phys. 42, 2634 (1965).

${ }^{9}$ M. K. Sen, Indian J. Phys. 10, 429 (1937).

${ }^{10}$ L. V. Gurvich, M. M. Novikov, and V. G. Ryabova, Opt. Spectrosc. 18, 68 (1965).

${ }^{11}$ B. R. Yadav, S. B. Rai, and D. K. Rai, Can. J. Phys. 57, 496 (1979).

${ }^{12}$ M. Pophristic, D. S. Pesic, A. AnticJovanovic, and V. Bojovic, Spectrosc. Lett. 29, 61 (1996).

${ }^{13}$ A. Anticjovanovic, V. D. Bojovic, and D. S. Pesic, Spectrosc. Lett. 27, 247 (1994).

${ }^{14}$ B. Vujisic, A. Antic-Jovanovic, V. Bojovic, D. S. Pesic, and M. Pophristic, J. Mol. Spectrosc. 193, 389 (1999).
${ }^{15}$ V. M. Kovba and I. A. Topol, J. Mol. Struct.: THEOCHEM 30, 65 (1986). ${ }^{16}$ I. D. Petsalakis, G. Theodorakopoulos, R. W. Gora, and S. Roszak, J. Mol. Struct.: THEOCHEM 672, 105 (2004).

${ }^{17}$ S. Gowtham, A. Costales, and R. Pandey, J. Phys. Chem. B 108, 17295 (2004).

${ }^{18}$ M. J. Zehe, D. A. Lynch, B. J. Kelsall, and K. D. Carlson, J. Phys. Chem. 83, 656 (1979).

${ }^{19}$ L. V. Serebrennikov, S. B. Osin, and A. A. Maltsev, J. Math. Phys. 81, 25 (1982).

${ }^{20}$ T. R. Burkholder, J. T. Yustein, and L. Andrews, J. Phys. Chem. 96, 10189 (1992).

${ }^{21}$ L. Andrews, G. P. Kushto, J. T. Yustein, E. Archibong, R. Sullivan, and J. Leszczynski, J. Phys. Chem. A 101, 9077 (1997).

${ }^{22}$ E. F. Archibong and A. St-Amant, Chem. Phys. Lett. 284, 331 (1998).

${ }^{23}$ Y. X. Bu, D. Z. Chan, and X. Y. Song, Int. J. Quantum Chem. 81, 222 (2001).

${ }^{24}$ R. B. Metz, A. Weaver, S. E. Bradforth, T. N. Kitsopoulos, and D. M. Neumark, J. Chem. Phys. 94, 1377 (1990).

${ }^{25}$ C. S. Xu, G. R. Burton, T. R. Taylor, and D. M. Neumark, J. Chem. Phys. 107, 3428 (1997).

${ }^{26}$ A. K. Wilson, D. E. Woon, K. A. Peterson, and T. H. Dunning, J. Chem. Phys. 110, 7667 (1999)

${ }^{27}$ T. H. Dunning, J. Chem. Phys. 90, 1007 (1989).

${ }^{28}$ M. J. Frisch, G. W. Trucks, H. B. Schlegel et al., Gaussian98, Revision A9 (Gaussian Inc., Pittsburgh, PA, 1998).

${ }^{29}$ S. R. Desai, H. B. Wu, and L. S. Wang, Int. J. Mass Spectrom. Ion Processes 159, 75 (1996).

${ }^{30}$ K. P. Huber and G. Herzberg, Molecular Spectra and Molecular Structure. IV. Constants of Diatomic Molecules (Van Nostrand Reinhold, New York, 1979).

${ }^{31}$ S. R. Desai, H. B. Wu, C. M. Rohlfing, and L. S. Wang, J. Chem. Phys. 106, 1309 (1997).

${ }^{32}$ A. V. Nemukhin and J. Almlof, J. Mol. Struct.: THEOCHEM 253, 101 (1992). 
The Journal of Chemical Physics is copyrighted by the American Institute of Physics (AIP). Redistribution of journal material is subject to the AIP online journal license and/or AIP copyright. For more information, see http://ojps.aip.org/jcpo/jcpcr/jsp 